oder Stäubchen, documentirten sie sich unter dem Mikroskop auch als die wohlausgebildetsten und für genanntes Alkaloïd charakteristisch'sten Krystalle, gleichfalls nur einen unberechenbar geringen Einfluss auszuüben im Stande sein.

\title{
Bericht über die neuesten, auf den Zusammenhang zwischen chemischer Zusammensetzung und physiolo- gischer Wirkung giftiger Substanzen beziiglichen Untersuchungen
}

von Dr. Hermann Köhler, Privatdocent zu Halle.

Ueber die Beziehungen zwischen chemischer Zusammensetzung und toxischer Wirkung giftiger Substanzen war bis vor Kurzem so gut wie gar nichts bekannt; nur Schroff's und Stahlschmidt's Untersuchungen über das Methylstrychnin machten hiervon eine Ausnahme. Mit Freude musste daher eine von $\mathrm{Crum}-\mathrm{Brown}$ und Fraser*) über diesen Gegenstand veröffentlichte Abhandlung, welche des Neuen viel enthält, begrüsst werden. Verf. glauben, dass Veränderungen der physiologischen Wirkung giftiger Substanzen besonders durch Verbindung derselben mit chemischen A tomcomplexen zu Stande kommen, und haben zuvörderst die durch Eintritt der Jodüre der Alkoholradicale in die stickstoffhaltigen chemischen Verbindungen, welche wir mit dem Namen der Alkaloïde zu bezeichnen pflegen, bedingten eingehend erforscht. Nach Ansicht der Verf. wird diese Addition im chemischen Sinne mit Verminderung der "Condensation, " d. h. unvollkommener Sättignng, z. B. des $\mathbf{N}$ in den Ammoniaken, worin er dreiwerthig auftritt, in Zusammenhang gebracht. Diese Abnahme der Condensation ist die, wenn auch nicht einzige, Bedingung für das Zustandekommen giftiger Wirkungen. Auf dem von Schroff betretenen Wege der Untersuchung weiter fortschreitend, stellten Verf. die Jodmethylverbindungen des Strychnins, Brucins,

*) Transactions of the Royal Society of Edinburgh. January. 6. the. 1868. - Crum-Brown and Fraser: Journal of anatomy and physiology II., 224. May 1868. 
Chem. Zusammensetz. u. phys. Wirkung gift. Substanzen etc. 101

Thebains, Kodeïns, Morphins und Nicotin's in folgender Weise dar.

Das feingepulverte Alkaloïd wird mit kohlensaurem Kali behandelt, ein Ueberschuss von in Weingeist gelöstem Jodmethyl zugegeben, und die Mischung, durch ihr doppeltes Volumen Spir. vini rectificatiss. verdünnt, 24 Stunden digeriren gelassen. Jetzt wird der Weingeist abdestillirt und die restirende Jodmethylverbindung umkrystallisirt. Da dieselbe in der Regel in Wasser löslich ist, so dient sie Verf. meistentheils auch zur Gewinnung der entsprechenden Schwefelsäureverbindung. Während nun $1 / 30-1 / 20$ Grn. Strychnin ein Kaninchen tödtet, wirken 12 Grn. Strychninmethyljodür gar nicht giftig, und liegt die lethale Dosis der Jodmethylverbindung bei 20 Grn. (!) Merkwürdigerweise riefen sowohl das Jodmethylstrychnin, als das gleichfalls untersuchte schwefelsaure Methylstrychnin bei den Versuchsthieren keinesweges die sich in Tetanus äussernden Erscheinungen der Strychnin-, sondern die in Lähmung der intermuskulären Nervenendignngen bei Intactheit der centripetalen Leitung (sensibl. Nerven) und des Herzens sich aussprechenden Symptome der Curarevergiftung hervor. Die Rücken - und Extremitätenmuskeln blieben hierbei schlaff, contractil und von stundenlang andauernder alkalischer Reaction. Durch die chemische Verbindung des Strychnins mit Jodmethyl wird also die ursprünglich auf das Rückenmark gerichtete, centrale Wirkung dieses Alkaloïdes dergestalt alienirt, dass nun die peripherischen, motorischen Nervenendigungen znerst angegriffen werden. Gleichzeitig wird aber auch die Intensität der Wirkung überhaupt um das 140 fache abgeschwächt.

Ganz ebenso verhielten sich Brucin-, Kodeïn-, Thebaïn-und Morphin-Methyljodür. Die lethale Dosis des schwefelsauren Methyl-Brucins liegt besonders hoch, diejenige des in Wasser leichter löslichen, entsprechenden Thebaïnsalzes dagegen niedriger; von letzterem waren 8 Gran nöthig, um den Tod von Kaninchen zu bewirken, während 1/5 Gran Thebaïn. pur. zn gleichem Zwecke ausreicht. Von der entsprechenden Kodeïnverbindung wurden 10 Grains, also die 12 fache Menge 
des reinen Alkaloïdes, nothwendig. Die physiologischen Wirkungen des Kodeïns wurden übrigens, indem das Alkoholradical in die Verbindung eintrat, nicht verändert. Die Mor phinverbind ung ist sehr schwer löslich; $20 \mathrm{Gr}$., subcutan injicirt, blieben völlig erfolglos, und während $8 \mathrm{Gr}$. Morphin per os beigebracht, zum Tode führten, erwiesen sich die auf die Höhe von $30 \mathrm{Gr}$. gesteigerten Gaben der Jodmethylverbindung als durchaus erfolglos. Versuche an Menschen bestätigten das über Morphin an Thieren Beobachtete in allen Punkten. Vier Grains des schwefelsauren Methylmorphins riefen bei Kaninchen Morphin-Narkose, aber keine Convulsionen hervor.

Das Jodmethylnicotin stellt leicht in kaltem Wasser lösliche Krystalle dar; fünf Grains konnten Kaninchen, ohne irgend welche Wirkung zu erzeugen, subcutan injicirt werden; frinfzehn Grains bedingten bedrohliche Vergiftungserscheinungen, aber erst 20 Grains waren tödtlich; Convulsionen blieben auch in diesem Falle ans.

Mit Jodmethyl selbst angestellte Versuche wiesen nach, das sich nicht etwa die Wirkungen desselben einfach mit denjenigen des ursprïnglich angewandten Alkaloïdes combiniren. Vielmehr wird, indem das Jodmethyl in die chemische Verbindung eintritt, die physiologische Wirkung der genannten Alkaloïde nicht nur wesentlich modificirt, sondern auch in so enormem Grade abgeschwächt, dass Verf. das Jodmethyl als Antidot der öfter bezeichneten giftigen Substanzen empfehlen zu dürfen glauben.

Die Beantwortung der letzteren Frage hat sodann W. B. Richardson beschäftigt. *) Er experimentirte mit Fröschen, Kaninchen und Meerschweinchen und bediente sich des Jodäthyls und Jodmethyls als Antidot der in Rede stehenden Alkaloïde, besonders des Stryehnins und Nicotins, in folgender Weise. Es wurden 3 Lösungen, nemlich

*) Abstract of Report to the British Association for the Advancement of Science 1868. 
Chem. Zusammensetz. u. phys. Wirkung gift. Substanzen etc. 103 Wasser,

b) von $1 / 30$ Gran Strychnin und 2 Minims Jodäthyl in eben soviel Weingeist und Wasser, und

c) von 2 Minims Jodäthyl in ebensoviel Weingeist und Wasser dargestellt. Ein Frosch bekam von Solut. a) injicirt und verfiel nach $1 \frac{1}{2}$ Minuten in Tetanus; ein $Z$ weiter bekam von Solut. b) und verhielt sich ebenso. Froseh Nr. 1 erhielt nun eine Lösung von fünf Minims Jodäthyl subcutan injicirt; nach zehn Minuten war der Tetanus vorüber und die abnorme Steigerung der Reflexerregbarkeit hatte abgenommen. Nach 20 Minuten war die Erschlaffung der Muskulatur vollständig und nur, wenn die Haut gereizt wurde, fanden sich leise Zuckungen derselben ein.

Frosch Nr. 2 wurde sodann 1 Grain Jodäthyl subcutan injicirt; sofort verschwand der Tetanus und machte completer Erschlaffung mit Erlöschen der Reflexerregbarkeit Platz. Am nächsten Morgen waren beide Frösche, ohne dass Aenderungen in ihrem Befinden eingetreten wären, todt.

Ein antidotarisches Verhalten des Jodäthyls dem Strychnin gegenüber war hieraus zwar ersichtlich geworden; da indess beide Frösche unter wesentlich verschiedenen Symptomen zu Grunde gegangen waren, so erschien eine nochmalige Wiederholung der Versuche geboten. Sie wurde vorgenommen wie folgt.

Frosch Nr. 3 erhielt erst eine Auflösung von 10 Minims reinen Jodäthyls ; einige Stunden danach befand er sich anscheinend bei unverändertem Wohlsein; dann aber wurden Zeichen vorhandener Motilitätslähmung bemerklich, und das Thier starb, nachdem es sich ganz so, wie Frosch Nr. 2 verhalten hatte, am nächsten Morgen. Hieraus ergab sich, dass bei Frosch Nr. 1 zu wenig, bei Frosch Nr. 2 zuviel Jodäthyl angewandt, dass Frosch Nr. 1 am Strychnin zu Grunde gegangen war, und, dass Jodäthyl an sich Frösche unter den bei Nr. 2 beschriebenen Erscheinungen zu tödten im Stande ist.

Es lag nun die Frage nahe, ob es möglich sei, das Verhältniss der ingerirten Strychnin- und Jodäthyldosen so 
genau zu treffen, dass die Wirkungen beider sich gegenseitig aufheben.

Dieses Verhältniss zu ermitteln ist Richardson für Strychnin und Jodmethyl sowenig, wie für Nicotin und die Jod- oder Bromverbindungen der Alkoholradicale gelungen. Wurden sehr grosse Alkaloïddosen angewandt, so gelang es zwar, das Leben der Thiere 24-28 Stunden zu fristen; dann aber trat regelmässig der Tod ein. Nun war schliesslich zu ermitteln, ob die Wirkungen der genannten Jodüre und Bromüre vorliegenden Falles chemische oder physiologi$\mathrm{sche}, \mathrm{d}, \mathrm{h}, \mathrm{ob}$ sie in einer chemischen Verbindung des Jodsalzes mit dem Alkaloïd, oder in der dic Alkaloïdwirkung compensirenden Wirkung des Jod- und Bromäthyls oder Methyls begründet waren. $\mathrm{Zu}$ diesem Behuf wurden anstatt der Jod - und Bromverbindungen die Nitrite der Alkoholradicale als Gegenmittel des Strychnins und Nicotins versucht. Der Erfolg war genau derselbe, als wären die Haloïdsalze angewandt worden; es gelang zwar, dem Strychnintetanus und den rom Nicotin bedingten Convulsionen dadurch vorzubeugen; niemals aber wurde der Punkt, wo Alkaloïd und Gegengift sich gewissermaassen in ihrer Wirkung neutralisiren, getroffen, und der Tod trat in allen Fällen ein. Es ist also nicht die durch den Eintritt der Alkoholverbindung mit Jod in das Alkaloïd gesetzte Veränderung in der chemischen Zusammensetzung des letzteren, welches die physiologischen Wirkungen desselben verändert, sondern die Aethylund Methylverbindungen erweisen sich (im Widerspruch mit Brown und Fraser) ihren physiologischen Eigenschaften und davon abhängigen Wirkungen nach, in gewissem Sinne als Antagonisten der Alkaloïde. Ein therapeutischer Gebrauch als Gegengift wird aber von den betreffenden Alkoholverbindungen in Fällen von Strychnin - und Nicotinvergiftung so lange nicht zu machen sein, als die Feststellung der zur gegenseitigen Aufhebung oder Neutralisation der Wirkungen der Alkaloïde sowohl; als der für kleine Thiere ebenfalls giftigen Jodverbindungen der Alkoholradicale nicht gelungen ist. 
Chem. Zusammensetz. u. phys. Wirkung gift. Substanzen etc. 105

Die neuesten, gleichfalls im höchsten Grade wichtigen Untersuchungen über denselben Gegenstand sind der französ. Akademie von Cahours und Jolyet*) mitgetheilt worden. Sie zeigten nemlich, dass, analog der durch Addition eines Alkoholradicals erzeugten, abweichenden Wirkung der pflanzlichen Alkaloïde, auch die Substitution eines solchen Radicales im Anilin ähnliche Differenzen in der Wirkung zur Folge hat. Während nemlich Anilindämpfe bei Fröschen Krämpfe verursachen, bewirken die des Methyl-, Aethyl- und Amylanilins nur Stupor und, bei stärkerer Einwirkung Aufhören der Reflexe und Athembewegung, während das Herz unverändert fortschlägt.

Ohne (wie dies Franzosen nicht selten passirt) von Brown's und Fraser's oben referirten Untersuchungen Kenntniss zu haben, bemerken die Verf. über Methyl- und Aethylstrychnin, dass sie wie Curare wirken, d. h. die intramuskulären Nerven lähmen, sensible Nerven und Herz dagegen intact lassen. (Für Methylstrychnin hat diese Thatsache schon Schroff constatirt). Nach Unterbindung der zuführenden Gefässe einer Extremität, treten in letzterer den bei Strychninvergiftung zu beobachtenden ähnliche, spontane Krämpfe auf, welche, weil sie nur nach Lähmung des intramuskulären Nervenausbreitungen zu Stande kommen, sonst nicht beobachtet werden. Nach $24-36$ Stunden hören diese Krämpfe auf und die Versuchsthiere erholen sich.

Um bei Warmblütern diese (von Brown und Fraser nicht erwähnten) langsam entstehenden und nur in langen, übrigens freien Intervallen auftretenden Krämpfe hervorzubringen, sind $20 \mathrm{Grm}$. Methyl- und 20-40 Grm. Aethylstrychnin, welche, per os applicirt, die Versuchsthiere nicht tödten, erforderlich.

Die jüngsten Berichte der Verf. beziehen sich auf A ethylconiin'und Diäthyleoniinjodür (Compt. rend. 1869. III. 149.). Coniin wirkt bekanntlich an sich dem Curare ähnlich

*) Comptes rendus de l'Académie. LVI. Nr. 22. p. 1131.1868. 
106 Chem. Zusammensetz. u. phys. Wirkung gift. Substanzen etc.

und erzengt u. a. klonische Krämpfe. Durch Eintritt der Aethylgruppe und des Jods in das Alkaloïd wird die Schnelligkeit der Wirkung dieses deswegen bekanntlich im höchsten Grade gefährlichen Giftes wesentlich herabgesetzt, und kommen die den Lähmungen bei warmblütigen Thieren vorangehenden Convulsionen bei Anwendung des mit Aethyl etc. verbundenen Conïns in Wegfall.

Dasselbe gilt von den durch Dr. Siewert hierselbst im Samen der $\mathrm{L}$ u p in en nachgewiesenen Coniinderivaten: Conydrin und Dimethylconydrin; sie bewirken sehr allmälig, und ohne zu Convulsionen zu führen, Motilitätslähmung und haben sonach Siewert's Beobachtungen die über die geringere Gefährlichkeit des Conydrins (dem Coniin gegenüber) von früheren Autoren gemachten Angaben durchaus bestätigt. Die Details seiner interessanten Untersuchungen über das Dimethylconydrin steht dieser fleissige Experimentator soeben im Begriff mitzutheilen. Seinen Veröffentlichungen vorzugreifen, kann nicht in unserer Absicht liegen, wohl aber dürfen wir hiermit die Gelegenheit ergreifen, auf die Resultate dieser mühevollen und nicht ungefährlichen Arbeit in Voraus aufmerksam zu machen. ${ }^{*}$ ) $H . K$.

*) Die in Obigem erwähnte Arbeit des Herrn Dr M. Siewert „,über die Alkaloïde der Lupinusaylen " findet sich jetzit abgedruekt im Mai-Juniheft 1869 der Zeitschrift für die gesammten Naturwissenschaften S. 426-463. Sie schliesst mit folgenden Ergebnissen:

1) In dem Bitterstoffe der Lupinen ist der Hauptmenge nach eine krystallisirbare Basis $\mathrm{C}^{20} \mathrm{H}^{21} \mathrm{NO}^{2}$ enthalten, welche bei $261^{\circ} \mathrm{C}$. siedet und kein vertretbares Wasserstoffatom enthält.

2) Es ist wahrscheinlich, dass der nicht krystallisirbare Theil des Bitterstoffs, der bei 306 bis $310^{\circ} \mathrm{C}$. siedet, aus einem Gemenge mehrer Bassen ( $\mathrm{C}^{14} \mathrm{H}^{15} \mathrm{NO}^{2}$ und $\left.\mathrm{C}^{16} \mathrm{H}^{17} \mathrm{NO}^{2}\right)$ besteht.

3) Ob kleine Mengen Coniin und Methylconydrin ursprünglich rorhanden sind, bleibt ungewiss.

H. $\boldsymbol{L}$. 